\title{
A sound field separation technique based on measurements with pressure-velocity probes
}

\author{
Zhang, Yong-Bin; Chen, Xin-Zhao; Jacobsen, Finn
}

Published in:

Journal of the Acoustical Society of America

Link to article, DOI:

$10.1121 / 1.3127128$

Publication date:

2009

Document Version

Publisher's PDF, also known as Version of record

Link back to DTU Orbit

Citation (APA):

Zhang, Y-B., Chen, X-Z., \& Jacobsen, F. (2009). A sound field separation technique based on measurements with pressure-velocity probes. Journal of the Acoustical Society of America, 125(6), 3518-3521.

https://doi.org/10.1121/1.3127128

\section{General rights}

Copyright and moral rights for the publications made accessible in the public portal are retained by the authors and/or other copyright owners and it is a condition of accessing publications that users recognise and abide by the legal requirements associated with these rights.

- Users may download and print one copy of any publication from the public portal for the purpose of private study or research.

- You may not further distribute the material or use it for any profit-making activity or commercial gain

- You may freely distribute the URL identifying the publication in the public portal

If you believe that this document breaches copyright please contact us providing details, and we will remove access to the work immediately and investigate your claim. 


\title{
A sound field separation technique based on measurements with pressure-velocity probes (L)
}

\author{
Yong-Bin Zhang a) and Xin-Zhao Chen \\ Institute of Sound and Vibration Research, Hefei University of Technology, Hefei 230009, China
}

\begin{abstract}
Finn Jacobsen
Department of Electrical Engineering, Acoustic Technology, Technical University of Denmark, Building 352, Ørsteds Plads, DK-2800 Kgs. Lyngby, Denmark
\end{abstract}

(Received 3 February 2009; revised 1 April 2009; accepted 10 April 2009)

\begin{abstract}
It has recently been shown that statistically optimized near field acoustic holography based on measurement with an array of pressure-velocity transducers makes it possible to distinguish between sources on the two sides of the array and thus suppress the influence of a disturbing source [F. Jacobsen and V. Jaud, J. Acoust. Soc. Am. 121, 1550-1558 (2007)]. However, the suggested technique uses a transfer matrix optimized for the source under test and may be expected to perform less well when the disturbing source is not placed symmetrically on the other side of the array, and this will usually be the case. In this letter, a modified method is presented.
\end{abstract}

(C) 2009 Acoustical Society of America. [DOI: 10.1121/1.3127128]

PACS number(s): 43.60.Sx, 43.60.Pt, 43.20.Rz [EGW] Pages: 3518-3521

\section{INTRODUCTION}

In conventional planar near field acoustic holography (NAH), it is a necessary condition that the sound sources are confined to half space (say, $z<0$ ), whereas the other half space is source-free and free-field conditions obtain. ${ }^{1,2}$ However, in many realistic situations, this condition cannot be satisfied because of reflections or disturbing sources. If the disturbing sources cannot be removed, a sound field separation technique should be applied to eliminate the influence of the disturbing sound. Such sound field separation techniques based on double layer pressure measurements have been investigated for years. ${ }^{3-6}$ An alternative single layer technique for separating the primary source from the disturbing source was recently proposed by Jacobsen and Jaud. ${ }^{7}$ This method is based on the statistically optimized version of NAH (SONAH) developed by Hald et al., ${ }^{8,9}$ and requires measurement of both the sound pressure and the normal component of the particle velocity in the measurement plane. Yet another method for separating sound field contributions using pressure-velocity measurement has been proposed by $\mathrm{Bi}$ and Chen. ${ }^{10}$ Their method combines the double layer technique with the equivalent source method, and the particle velocity is obtained from a finite difference approximation.

The transfer matrices employed by Jacobsen and Jaud's method have been optimized for the primary source. ${ }^{7}$ Thus one might perhaps expect a reduced performance when the primary source and the disturbing source are not located symmetrically with respect to the measurement plane, as anticipated in Ref. 7. The purpose of this letter is to examine the matter and present an improvement to the method that extends it to the general case where the location of the disturbing source is arbitrary on the side of the measurement

\footnotetext{
a) Author to whom correspondence should be addressed. Electronic mail: zybmy1997@163.com
}

plane opposite to that of the primary source. The modified method is compared with the original method in a simulation study as well as experimentally.

\section{THE SINGLE LAYER METHOD AND A MORE GENERAL VERSION}

The so-called $p-u$ method described in Ref. 7 combines a pressure-based SONAH prediction with a particle velocitybased prediction, the idea being that the two estimates should be identical if the sound is coming from the primary source but have an opposite sign if the sound is coming from a disturbing source located directly opposite to the measurement plane from the primary source because of the fact that the particle velocity is a vector whereas the pressure is a scalar. Thus the sound pressure in a grid of $N$ points $\left(\mathbf{r}_{s, n}\right)$ in the prediction plane $\left(z=z_{s}\right)$ generated only by the primary source can be expressed as the average of a weighted sum of measured pressure and particle velocity signals measured at $N$ positions $\left(\mathbf{r}_{h, n}\right)$ in the hologram plane $\left(z=z_{h}\right)$,

$$
\mathbf{p}_{1}^{T}\left(\mathbf{r}_{s}\right)=\frac{1}{2}\left[\mathbf{p}^{T}\left(\mathbf{r}_{h}\right) \mathbf{C}_{p p}+\mathbf{u}_{z}^{T}\left(\mathbf{r}_{h}\right) \mathbf{C}_{v p}\right]
$$

where $T$ denotes matrix transpose, $\mathbf{p}_{1}\left(\mathbf{r}_{s}\right)$ is a column vector with the predicted pressures due to the primary source, and $\mathbf{p}\left(\mathbf{r}_{h}\right)$ and $\mathbf{u}_{z}\left(\mathbf{r}_{h}\right)$ are column vectors with the measured pressures and particle velocities generated both by the primary source and the disturbing source. The two $N \times N$ transfer matrices $\mathbf{C}_{p p}$ and $\mathbf{C}_{v p}$ are determined by requiring that an infinite set of propagating and evanescent elementary waves of the form

$$
\Phi_{m}(\mathbf{r})=e^{-j\left(k_{x, m} x+k_{y, m} y+k_{z, m} z\right)}, \quad m=1,2, \ldots, M, \quad M \rightarrow \infty,
$$

originating from the primary source plane, satisfy Eq. (1) with optimal accuracy (with the $e^{j \omega t}$ sign convention). This implies that 


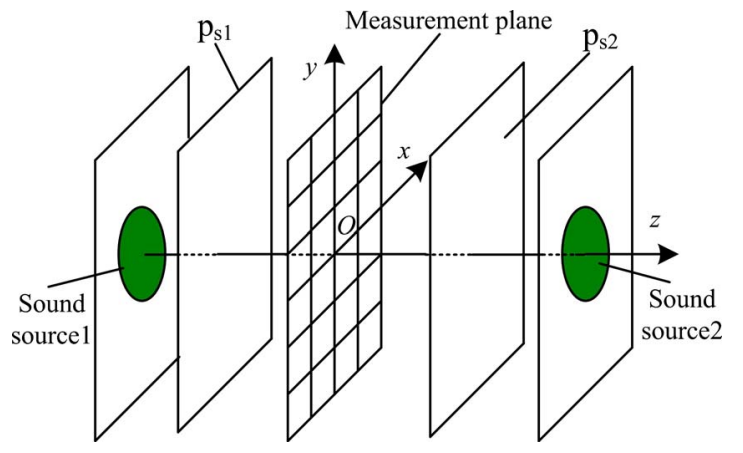

FIG. 1. (Color online) Configuration.

$$
\mathbf{B}=\mathbf{A C}_{p p}, \quad[\mathbf{A}]_{m n}=\Phi_{m}\left(\mathbf{r}_{h, n}\right), \quad[\mathbf{B}]_{m n}=\Phi_{m}\left(\mathbf{r}_{s, n}\right),
$$

and that

$$
-j \omega \rho \int \mathbf{B} d z_{s}=\mathbf{A C}_{v p}
$$

from which the two transfer matrices are determined; see Refs. 7-9.

A modified version of this method is described in what follows. The configuration is shown in Fig. 1, where $\mathbf{p}_{s 1}$ is the sound pressure generated only by the primary source in the prediction plane, which may coincide with the primary source plane or may be between the source and the measurement plane, and $\mathbf{p}_{s 2}$ is the sound pressure generated only by the disturbing source on the other side of the measurement plane.

The pressure and the particle velocity generated by both sources in the measurement plane can be written as sums of two contributions:

$$
\begin{aligned}
& \mathbf{p}^{T}\left(\mathbf{r}_{h}\right)=\mathbf{p}_{s 1}^{T} \mathbf{C}_{p p 1}+\mathbf{p}_{s 2}^{T} \mathbf{C}_{p p 2}, \\
& \mathbf{u}_{z}^{T}\left(\mathbf{r}_{h}\right)=\mathbf{p}_{s 1}^{T} \mathbf{C}_{p v 1}-\mathbf{p}_{s 2}^{T} \mathbf{C}_{p v 2},
\end{aligned}
$$

where $\mathbf{C}_{p p 1}$ and $\mathbf{C}_{p v 1}$ are determined from

$$
\mathbf{A}=\mathbf{B C}_{p p 1}
$$

and

$$
(-1 /(j \omega \rho))\left(\partial \mathbf{A} / \partial z_{h}\right)=\mathbf{B C}_{p v 1} .
$$

However, the transfer matrices for the disturbing source are different. They should be optimized for elementary waves coming from the disturbing source, and therefore they should be determined by another set of propagating and evanescent elementary waves:

$$
\Psi_{m}(\mathbf{r})=e^{-j\left(k_{x, m} x+k_{y, m} y-k_{z, m} z\right)}, \quad m=1,2, \ldots, M, \quad M \rightarrow \infty .
$$

The two transfer matrices $\mathbf{C}_{p p 2}$ and $\mathbf{C}_{p v 2}$ can now be determined in the same way as Eqs. (7) and (8) by replacing $\mathbf{A}$ and $\mathbf{B}$ with $[\mathbf{A}]_{m n}=\Psi_{m}\left(\mathbf{r}_{h, n}\right)$ and $[\mathbf{B}]_{m n}=\Psi_{m}\left(\mathbf{r}_{s, n}\right)$. Finally, combining Eqs. (5) and (6) gives the pressure generated by the primary source,

$$
\mathbf{p}_{s 1}^{T}=\left[\mathbf{u}_{z}^{T}\left(\mathbf{r}_{h}\right)+\mathbf{p}^{T}\left(\mathbf{r}_{h}\right) \mathbf{C}_{p p 2}^{-1} \mathbf{C}_{p v 2}\right]\left(\mathbf{C}_{p v 1}+\mathbf{C}_{p p 1} \mathbf{C}_{p p 2}^{-1} \mathbf{C}_{p v 2}\right)^{-1} .
$$

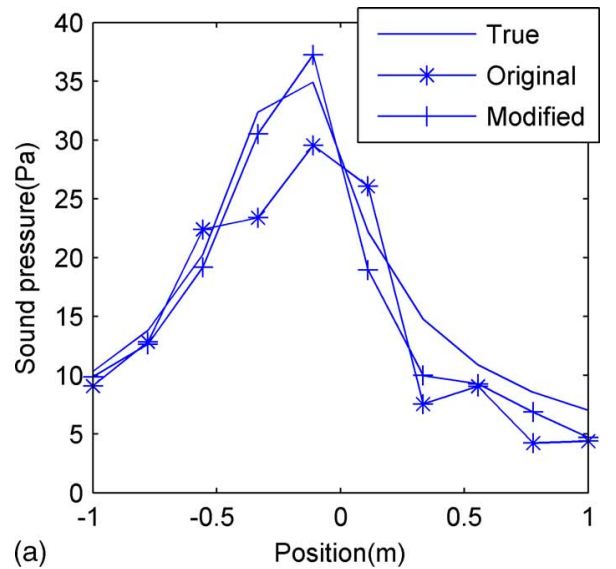

(a)

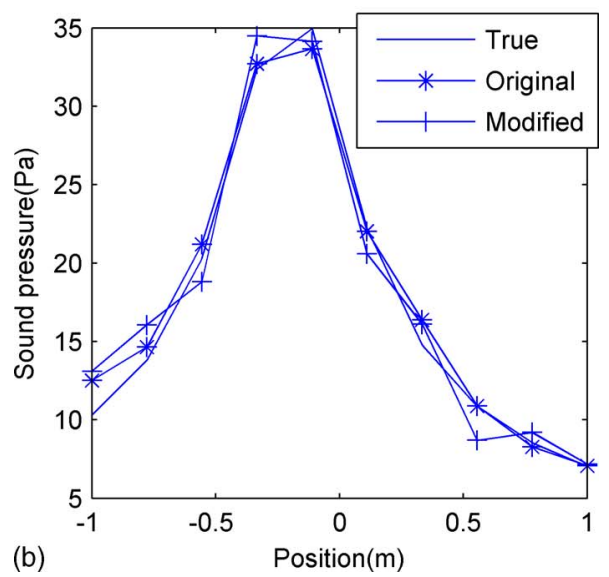

FIG. 2. (Color online) True and reconstructed undisturbed pressure along the $x$-axis at $500 \mathrm{~Hz}$ with the primary and secondary source placed nonsymmetrically (a) and placed symmetrically (b).

It should be mentioned that the Tikhonov regularization is needed when all transfer matrices are computed.

\section{A SIMULATION STUDY}

To examine the performance of the modified method, a simulation study has been carried out. A test case with two monopoles not located at symmetrical positions was examined. One monopole was regarded as the primary source and placed at $(-0.2,0,-0.2)(x, y, z$, coordinates in $\mathrm{m})$, and the other one was regarded as the disturbing source and placed at $(0.2,0,0.1)$ on the other side of the measurement plane, which was located at $z=0$. The sound pressure and normal component of the particle velocity were "measured" in a grid of $10 \times 10$ points with dimensions $2 \times 2 \mathrm{~m}^{2}$, and noise was added to the measured data corresponding to a signal-tonoise ratio of $20 \mathrm{~dB}$.

Figure 2(a) shows the "true" undisturbed sound pressure at $500 \mathrm{~Hz}$ along the $x$-axis in the measurement plane, and the reconstructed undisturbed pressure in the same plane determined using the original $p-u$ method based on Eq. (1) and the new version based on Eq. (10). It can be seen that the new version of the $p-u$ method gives much better results than the original version. Other test cases (not shown) have confirmed this observation.

Figure 2(b) shows the effect of moving the disturbing source to the position $(-0.2,0,0.2)$, which is symmetrical 

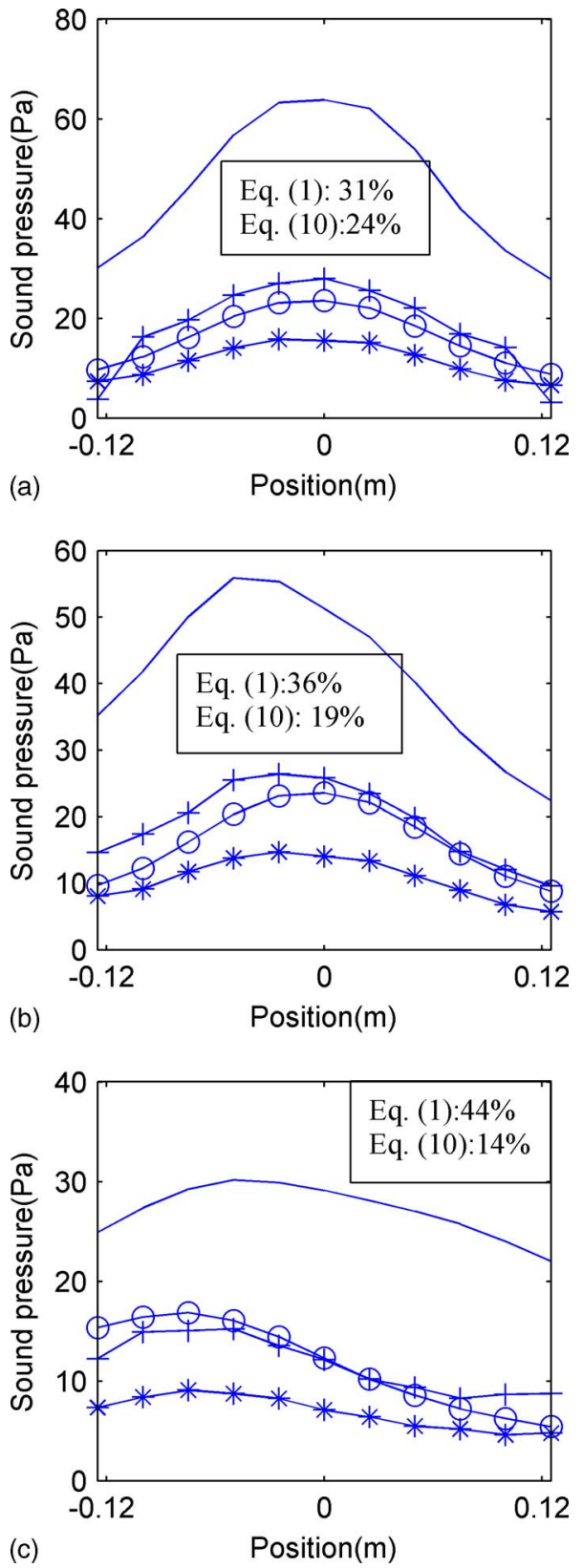

FIG. 3. (Color online) True and reconstructed pressure along the $x$-axis at $320 \mathrm{~Hz}$ in case 1 (a), case 2 (b), and case 3 (c). Solid line: total pressure; line with circles: true pressure generated by the primary source; line with crosses: reconstruction based on Eq. (10); line with stars: reconstruction based on Eq. (1). The global errors are also given.

with respect to the measurement plane. Not surprisingly, the original $p-u$ method performs very well in this case, in fact, even slightly better than the alternative method.

\section{EXPERIMENTAL RESULTS}

An experiment has been carried out in a large anechoic room at the Technical University of Denmark. A loudspeaker mounted in a sphere and an "experimental monopole" served as the primary source and the disturbing source, respectively. The latter device, described in Ref. 11, consists of a tube driven by a loudspeaker. The sound pressure and the particle velocity were measured at $11 \times 11$ points with a single $1 / 2$ in. $p-u$ sound intensity probe produced by Microflown. The
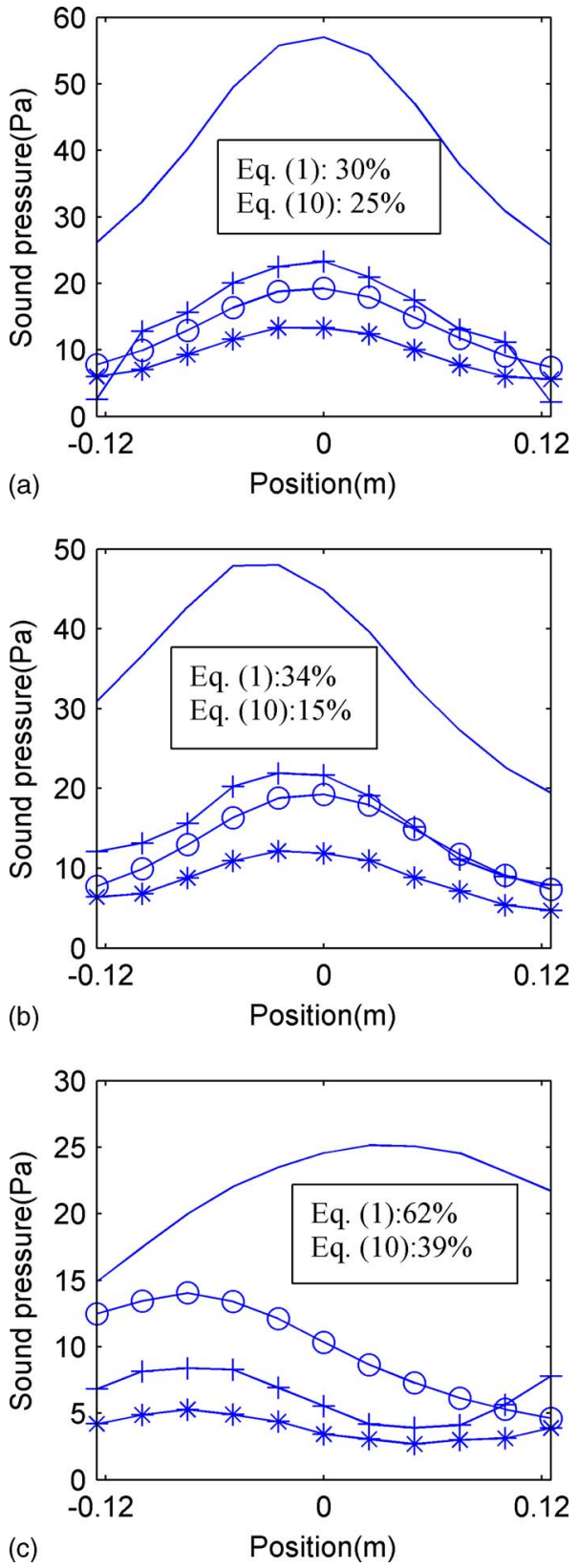

FIG. 4. (Color online) As in Fig. 3 but at $528 \mathrm{~Hz}$.

measurement plane with dimensions $25 \times 25 \mathrm{~cm}^{2}$ was located at $z=0$, and the measurement was carried out with the two sources operating together and with only the primary source turned on. This plane also served as prediction plane. Three test cases were examined: a symmetrical case (1) with the primary source at $(0,0,-0.05)$ (coordinates in $\mathrm{m})$ and the disturbing source at $(0,0,0.05)$, a nonsymmetrical case (2) with the primary source at $(0,0,-0.05)$ and the disturbing source at $(-0.05,0.05,0.05)$, and yet another nonsymmetrical case (3) with the primary source at $(-0.075,0,-0.08)$ and the disturbing source at $(0.075,0.05,0.14)$.

Figures 3-5 show the pressure generated by two sources together, the undisturbed pressure generated by the primary source, reconstructed using the method based on Eq. (1), and reconstructed using the modified method based on Eq. (10), and relative errors between the undisturbed pressure and the reconstructed pressure obtained by Eqs. (1) and (10), at three 

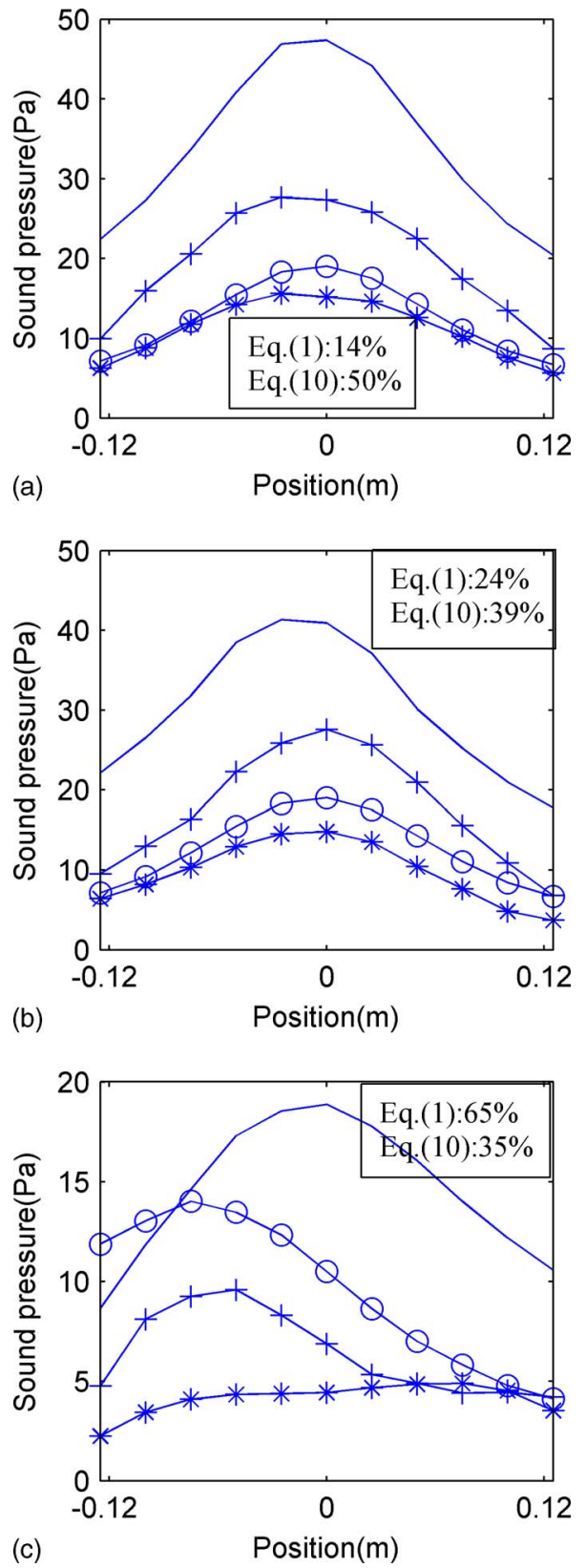

FIG. 5. (Color online) As in Fig. 3 but at $1016 \mathrm{~Hz}$.

different frequencies, 320, 528, and $1016 \mathrm{~Hz}$, respectively. In the symmetrical case 1 , both methods perform reasonably well, although the original method is clearly better than the modified method at $1016 \mathrm{~Hz}$. However, in the two nonsymmetrical cases (2 and 3), the method based on Eq. (10) is somewhat better than the original method based on Eq. (1), in particular, at low frequencies. It should finally be mentioned that the reconstructed phase has also been examined. The results, not shown, showed a similar tendency.

\section{CONCLUSIONS}

The single layer $p-u$ method proposed by Jacobsen and Jaud can separate sound field contributions coming from the two sides of the measurement plane and performs well in some cases. However, the primary source and the disturbing source are usually not located symmetrically, and in such cases the alternative version described and examined in this letter somewhat performs better.

\section{ACKNOWLEDGMENT}

The authors would like to thank Microflown for lending us a $p$ - $u$ sound intensity probe. This work was supported by the National Natural Science Foundation of China (Grant Nos. 50675056 and 10874037) and Fok Ying Tung Education Foundation (Grant No. 111058). Additionally, the China Scholarship Council is acknowledged for financial support.

${ }^{1}$ J. D. Maynard, E. G. Williams, and Y. Lee, "Nearfield acoustic holography: I. Theory of generalized holography and the development of NAH," J. Acoust. Soc. Am. 78, 1395-1413 (1985).

${ }^{2}$ W. A. Veronesi and J. D. Maynard, "Nearfield acoustic holography (NAH): II. Holographic reconstruction algorithms and computer implementation," J. Acoust. Soc. Am. 81, 1307-1322 (1987).

${ }^{3}$ G. V. Frisk, A. V. Oppenheim, and D. R. Martinez, "A technique for measuring the plane-wave reflection coefficient of the ocean bottom," J. Acoust. Soc. Am. 68, 602-612 (1980).

${ }^{4}$ M. T. Cheng, J. A. Mann III, and A. Pate, "Wave-number domain separation of the incident and scattered sound field in Cartesian and cylindrical coordinates,” J. Acoust. Soc. Am. 97, 2293-2303 (1995).

${ }^{5}$ J. Hald, "Patch holography in cabin environments using a two-layer handheld array with an extended SONAH algorithm," in Proceedings of Euronoise 2006, Tampere, Finland (2006).

${ }^{6}$ C.-X. Bi, X.-Z. Chen, and J. Chen, "Sound field separation technique based on equivalent source method and its application in nearfield acoustic holography," J. Acoust. Soc. Am. 123, 1472-1478 (2008).

${ }^{7}$ F. Jacobsen and V. Jaud, "Statistically optimized near field acoustic holography using an array of pressure-velocity probe," J. Acoust. Soc. Am. 121, 1550-1558 (2007).

${ }^{8}$ R. Steiner and J. Hald, "Near-field acoustical holography without the errors and limitations caused by the use of spatial DFT," Int. J. Acoust. Vib. 6, 83-89 (2001).

${ }^{9}$ Y. T. Cho, J. S. Bolton, and J. Hald, "Source visualization by using statistically optimized nearfield acoustical holography in cylindrical coordinates," J. Acoust. Soc. Am. 118, 2355-2364 (2005).

${ }^{10} \mathrm{C} . \mathrm{X}$. Bi and X.-Z. Chen, "Sound field separation technique based on equivalent source method using pressure-velocity measurements and its application in nearfield acoustic holography," in Proceedings of InterNoise, Shanghai, China (2008).

${ }^{11}$ S. Gade, N. Møller, J. Hald, and L. Alkestrup, "The use of volume velocity source in transfer measurements," in Proceedings of Inter-Noise 2004, Prague, Czech Republic (2004). 